\title{
Elastin-like polypeptide fusion for precision design of protein-polymer conjugates with improved pharmacology
}

\author{
Youqing Shen
}

Therapeutic proteins and peptides are characteristic by their high biological activity and specificity, but their clinical uses are bottlenecked by their poor stability, short in vivo half-life and immunogenicity [1]. One typical example is recombinant human interferon alpha (IFN- $\alpha$ ), FDA-approved and widely used in treatments of viral diseases and cancer, yet its short plasma half-life $\left(t_{1 / 2}=2-4 \mathrm{~h}\right)$ necessitates frequent dosing to achieve sustained efficacy, causing adverse side effects and poor patient compliance [2].

Conjugating poly(ethylene glycol) (PEG) to proteins, called PEGylation, is an effective approach to solve this problem. For instance, IFN- $\alpha$ conjugated with $40 \mathrm{kDa}$ branched PEG (PEGASYS, $t_{1 / 2}=77 \mathrm{~h}$ ) or $20 \mathrm{kDa}$ linear PEG (PEGINTRON, $t_{1 / 2}=35 \mathrm{~h}$ ) shows superior pharmacokinetics compared with native IFN- $\alpha$ [3]. However, PEGylation suffers from three major problems: 1) PEG chains are attached to sites randomly on the protein surfaces through the reactive amino acid residues, producing heterogeneous products of positional isomers with reduced activity. 2) Purification and isolation of these positional isomers are difficult [4]. 3) The reaction involves two large macromolecules and thus results in low yields $(<10 \%)$.

Albufusion, genetic fusion of proteins to human serum albumin (HSA), is an alternative strategy $[5,6]$. However, because of the large bulky size of HSA, IFN- $\alpha 2 b$ and HSA fusion protein (Albinterferon) had only about $1 \%$ of the in vitro bioactivity of native IFN-a2b [7]. Moreover, Albinterferon did not show superiority to PEGylated IFN- $\alpha$ in a randomized trial for treatment of patients with chronic hepatitis $\mathrm{C}$ virus $[8,9]$.

Therefore, in the past decades people have been dreaming of a revolutionary protein conjugation strategy with high precisity, yields and activity as well as significantly improved pharmacological profiles.

In a recent online publication of Advanced Materials,
Professor Weiping Gao from Tsinghua University, reports such a methodology, ELPfusion (Fig. 1) [10]. For the first time, they have demonstrated C-terminal fusion of IFN-a to an elastin-like polypeptide (ELP) to form a well-defined IFN-ELP fusion protein that was long acting and highly potent for cancer therapy. IFN-ELP fusion protein can be easily produced in $E$. coli with high yield and rapidly purified by a facile chromatography-free purification protocol of inverse transition cycling (ITC). Notably, the IFN-ELP fusion protein had much higher activity retention (41.1\%) than PEGylated IFN- $\alpha$ (7\%) and Albinterferon (1\%). Moreover, IFN-ELP fusion protein possessed a 27.7-fold longer circulating half-life $(8.6 \mathrm{~h})$ than IFN- $\alpha(0.3 \mathrm{~h})$ and dozens of times more tumor accumulation than IFN- $\alpha$. More interestingly, the fusion protein almost completely inhibited tumor growth without apparent toxicity, while IFN-a had little inhibition effect on tumor growth. These findings may pave the way for the treatment of cancer and potentially viral diseases with IFN-ELP fusion proteins.

Therefore, ELPfusion provides a solution to decadeslong challenge in protein-polymer conjugation. It may accelerate the development of more and better protein conjugates for therapeutics of a variety of diseases in the future.

Received 16 October 2015; accepted 20 October 2015; published online 23 October 2015

1 Veronese FM. Peptide and protein PEGylation: a review of problems and solutions. Biomaterials, 2001, 22: 405-417

2 Bailon P, Palleroni A, Schaffer CA, et al. Rational design of a potent, long-lasting form of interferon: a $40 \mathrm{kDa}$ branched polyethylene glycol-conjugated interferon $\alpha-2 a$ for the treatment of hepatitis C. Bioconjugate Chem, 2001, 12: 195-202

3 Zeuzem S, Feinman SV, Rasenack J, et al. Peginterferon alfa-2a in patients with chronic hepatitis C. N Engl J Med, 2000, 343: 16661672

4 Zhao W, Liu F, Chen Y, et al. Synthesis of well-defined proteinpolymer conjugates for biomedicine. Polymer, 2015, 66: A1-A10

5 Subramanian GM, Fiscella M, Smith AL, et al. Albinterferon $\alpha-2 b$ : a

Center for Bionanoengineering \& Key Laboratory of Biomass Chemical Engineering of Ministry of Education, Department of Chemical and Biological Engineering, Zhejiang University, Hangzhou 310027, China Email: shenyq@zju.edu.cn 

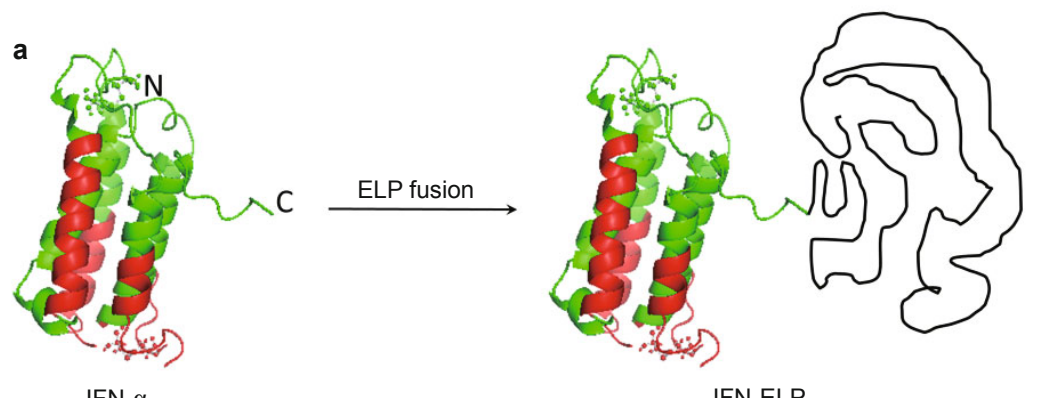

IFN-ELP
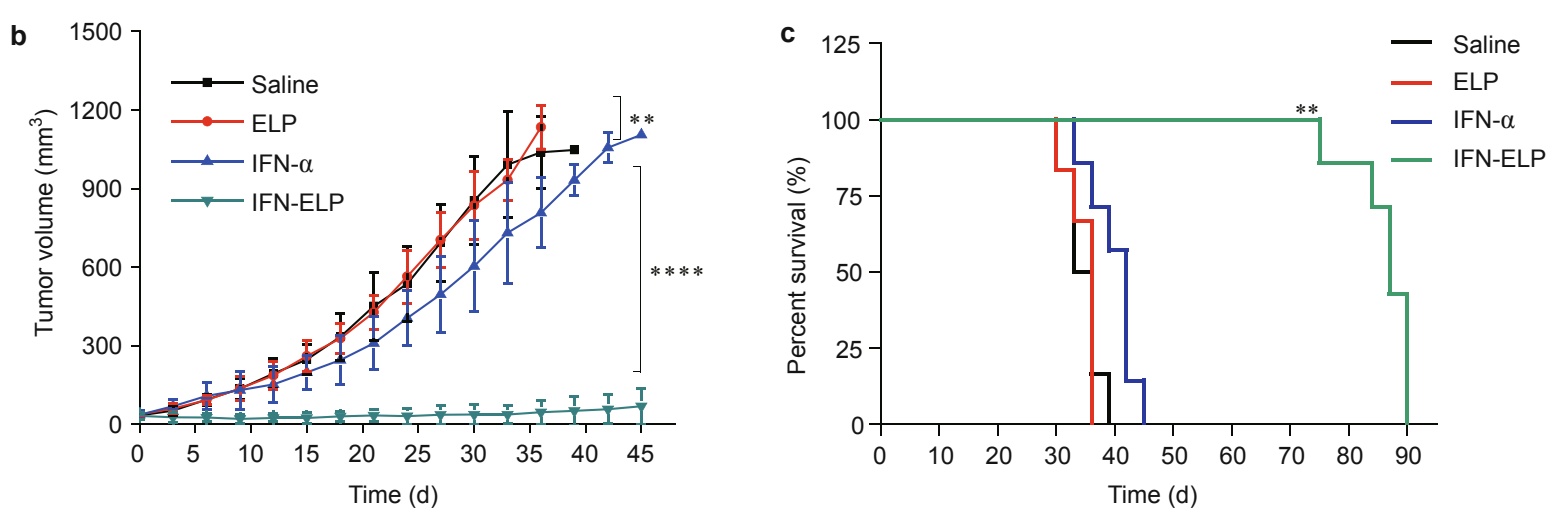

Figure 1 In vivo antitumor efficacy of IFN-ELP. (a) Synthesis of IFN-ELP by ELPfusion. (b) Inhibition of tumor growth post-treatment. (c) Cumulative survival of mice. Reprinted with permission from Ref. [10], Copyright 2015, Wiley-VCH Verlag GmbH \& Co. KGaA.

genetic fusion protein for the treatment of chronic hepatitis C. Nat Biotechnol, 2007, 25: 1411-1419

6 Zhao HL, Xue C, Du JL, et al. Balancing the pharmacokinetics and pharmacodynamics of interferon- $\alpha 2 b$ and human serum albumin fusion protein by proteolytic or reductive cleavage increases its in vivo therapeutic efficacy. Mol Pharmaceutics, 2012, 9: 664-670

7 Huang YS, Chen Z, Yang ZY, et al. Preparation and characterization of a potent, long-lasting recombinant human serum albumininterferon-alpha $2 \mathrm{~b}$ fusion protein expressed in Pichia pastoris. Eur J Pharm Biopharm, 2007, 67: 301-308

8 Zeuzem S, Sulkowski MS, Lawitz EJ, et al. Albinterferon alfa-2b was not inferior to pegylated interferon- $\alpha$ in a randomized trial of patients with chronic hepatitis C virus genotype 1. Gastroenterology, 2010, 139: 1257-1266

9 Nelson DR, Benhamou Y, Chuang WL, et al. Albinterferon alfa-2b was not inferior to pegylated interferon- $\alpha$ in a randomized trial of patients with chronic hepatitis $\mathrm{C}$ virus genotype 2 or 3 . Gastroenterology, 2010, 139: 1267-1276

$10 \mathrm{Hu}$ J, Wang GL, Liu XY, et al. Enhancing pharmacokinetics, tumor accumulation, and antitumor efficacy by elastin-like polypeptide fusion of interferon alpha. Adv Mater, 2015, doi: 10.1002/ adma.201503440 